\title{
Field Emission In-Lens Scanning Electron Microscopy of the Nuclear Envelope and Other Cellular Structures.
}

\author{
Martin W. Goldberg ${ }^{1}$, Jindřiška Fišerová ${ }^{2}$, Terence D. Allen ${ }^{3}$ \\ 1. School of Biological and Biomedical Sciences, Durham University, Science Laboratories, \\ South Road Durham, DH1 3LE. UK \\ 2. Institute of Molecular Genetics of the ASCR, v. v. i., Videnska 1083, 14220 Prague 4, Czech \\ Republic \\ 3. The Institute of Cancer Studies, University of Manchester, UK
}

Field emission in-lens scanning electron microscopes (FEISEMs) can achieve sub-nanometer resolution. This is the level of biological macromolecules and macromolecular complexes. Such instruments can therefore be used to study the surface structure of sub-cellular components, showing details of the organization of proteins and other macromolecules within these structures. Biological material, however, has a high water content and needs to be dried or frozen to be compatible with the vacuum within the microscope. It is electrically non conductive and therefore needs to be infiltrated, or coated, with metal to avoid problems with charge build up, causing imaging artifacts and instability. Most surfaces are enclosed within membrane bound compartments and are therefore inaccessible to surface imaging techniques such as FEISEM. Many of the membrane surfaces are fragile and can be damaged or altered during fixation drying and exposure to the intense beam of electrons in the microscope. Therefore to get useful information by FEISEM, care and consideration has to be given to the steps of sample preparation, and imaging parameters (e.g. current and voltage). Cryo methods have proved useful to tackle several of these problems. Cryo-fracturing/planing/sectioning is useful to expose surfaces of interest, whereas the use of a cryo-stage to image frozen hydrated samples within the microscope can prevent problems with fixation and drying artifacts. Work at room temperature on chromosome structure and banding used conductive staining involving thiocarbohydrazide and Osmium tetroxide [1], giving good structural detail without metal coating. However this results in a high level of backscatter electrons and hence SEIIs, creating noise. This proved unsuitable for imaging the nuclear membranes and nuclear pore complex (NPC) at the periphery of the nucleus overlying dense chromatin. Protocols [2] using tannic acid to stabilize filamentous components of the NPC (Fig. 1), such as the NPC basket (fishtrap), were an important development. However, development of high resolution metal coats [3], to provide signal solely from the surface, was pivotal to enable us to image fine structural detail of the NPC [4] and nuclear lamina [5]. Suitable coats can be obtained by sputter coating several refractory metals. However chromium is particularly useful because of its relatively low atomic number compared to gold. Gold nano-particles can be used to tag antibodies. A backscatter detector can be used to distinguish the gold particle from chromium coat, unequivocally identifying the epitope position. To image a structure such as the inner nuclear membrane requires the surface to be exposed. This can be achieved by isolating Xenopus oocyte nuclear envelopes. However, the inner nuclear membrane of most cells is tightly associated with the chromatin and other intra-nuclear structures. Therefore we developed simple fracturing methods [6] to remove the overlying material, exposing the inner membrane and allowing us, for instance, to show that plants possess a lamina-like structure and NPC baskets [7]. Alternatively samples can be frozen and fractured or sectioned. Such samples can be imaged directly using a cryo-SEM stage. Generally this 
requires samples to be freeze-etched, then metal coated at low temperature. Although routine in a dedicated cryo-SEM, this is challenging in an in-lens SEM where the coating and etching has to be done in a separate coating unit, necessitating transfer between this and SEM. A more straightforward alternative is to thaw the fractured/cryo-sectioned samples into fix, and dry, coat and image at room temperature. FEISEM can also be used to study the organization of cytoskeletal filaments, and clathrin coated pits and vesicles after detergent extraction and careful fixation (Fig.2). [8].

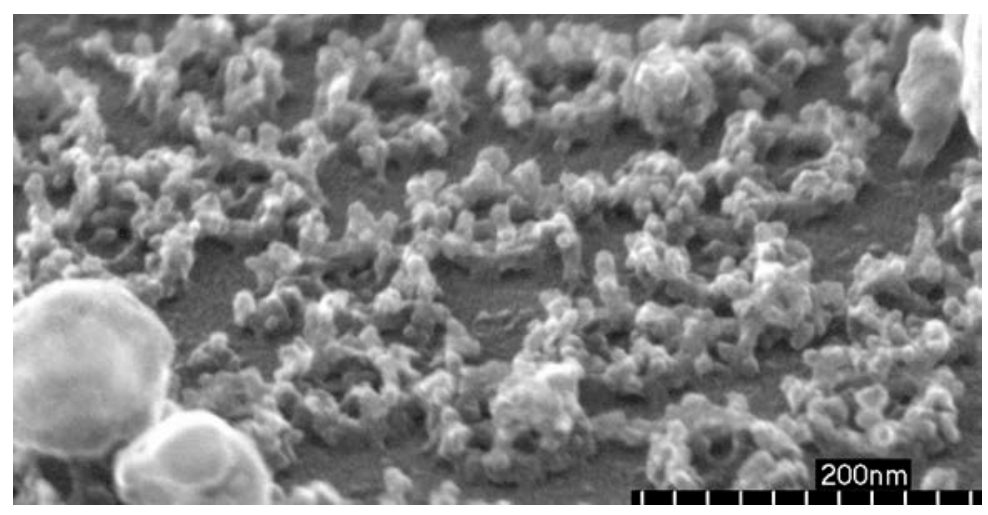

Fig. 1. FEISEM image of the cytoplasmic face of Xenopus oocyte nuclear envelope tilted 30 degrees.

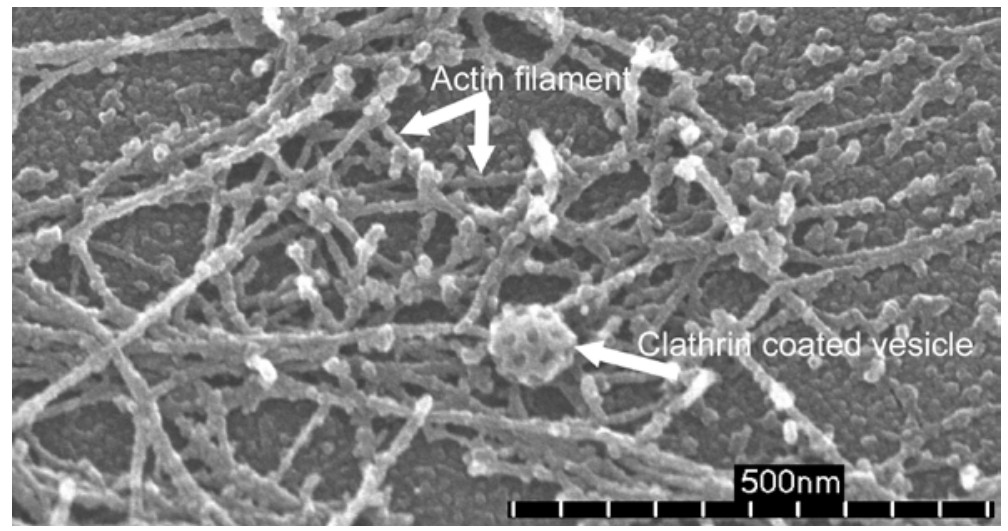

Fig. 2. Periphery of HaCaT cell after extraction with $0.5 \%$ Triton X100 showing actin filaments and clathrin coated vesicle.

References

[1] CJ Harrison et al, J Cell Sci 56 (1982), p409.

[2] H Ris, Electron Microsc Soc Am Bull 21 (1991), p54

[3] RP Apkarian, Scanning Microsc 8 (1994)289.

[4] MW Goldberg and TD Allen, J Mol Bio. 257 (1996), p848.

[5] MW Goldberg et al, J Cell Sci 121 (2008), p215.

[6] TD Allen et al, Nature Protocols 2 (2007), p1180.

[7] J Fiserova, E Kiseleva and MW Goldberg, Plant J 59 (2009), p243.

[8] Thanks to S Rutherford, G Bennion, C Richardson and H Grindley for technical assistance. 